\title{
Cryogenically cooled 946nm Nd:YAG laser
}

\author{
SungJin Yoon and Jacob I. Mackenzie \\ Optoelectronics Research Centre, University of Southampton, Southampton, SO17 1BJ, UK \\ sjy1g12@orc.soton.ac.uk
}

\begin{abstract}
We present the first multi-Watt demonstration of a diode pumped cryogenically cooled $\mathrm{Nd}$ :YAG operating on the $946 \mathrm{~nm},{ }^{4} \mathrm{~F}_{3 / 2} \rightarrow{ }^{4} \mathrm{I}_{9 / 2}$ transition. Preliminary results at LN temperature produced $3.8 \mathrm{~W}$ of output power for $20.7 \mathrm{~W}$ of incident pump power and a slope efficiency of $28 \%$. OCIS codes: (140.3530) Lasers, neodymium; (140.3480) Lasers, diode-pumped; (140.3580) Lasers, solid-state.
\end{abstract}

\section{Introduction}

Lasers operating in the 0.95 micron wavelength region are of particular interest for measurement of atmospheric water vapour by Differential Absorption LIDAR, or second harmonic frequency conversion targeting the main transmission window of water. Ideally these systems require higher energy and average powers than are currently available. There are three main drawbacks for power scaling this laser system, firstly it's weak effective stimulated emission cross section, $\sigma_{\mathrm{e}} \sim 4 \mathrm{pm}^{2}$, second, the requirement for high pump-intensity to reach threshold, in part due to reabsorption losses but also the low gain characteristics, which in turn implies a high thermal-load-density and associated thermo-optical aberrations, and third, significant competition with the stronger four level transition around $1.06 \mu \mathrm{m}$. Cryogenic cooling has proven extremely beneficial for power-scaling quasi-three-level Ytterbium laser systems, capitalizing on the reduction in reabsorption losses, increase in emission cross section, and more importantly, orders of magnitude improvement in the thermo-optical properties of the host media [1-3]. Despite this little effort has been attributed to Neodymium-doped materials, presumably due to the higher quantum defect heating and the already decent performance of the four-level $1064 \mathrm{~nm}$ transition. Nonetheless the potential for higher power operation of Nd:YAG quasi-four-level transition appears unexplored, and quite rich, noting that two of the three limiting parameters for room-temperature operation will be drastically improved by cooling the active medium to cryogenic temperatures, namely increased gain and reduced thermo-optic degradation.

In this paper we have investigated for the first time, to the best of our knowledge, multi-Watt laser performance of the $946 \mathrm{~nm}^{4} \mathrm{~F}_{3 / 2} \rightarrow{ }^{4} \mathrm{I}_{9 / 2}$ transition of $\mathrm{Nd}$ :YAG when cryogenically cooled by liquid nitrogen (LN) in an opticaldewar. In these preliminary results $3.8 \mathrm{~W}$ of output power was obtained with $20.7 \mathrm{~W}$ of incident power, giving a slope efficiency of $28 \%$ with respect to the incident pump power.

\section{Theory}

The theoretical performance of the quasi-four-level Nd:YAG laser transition has been well documented in the literature since the first reports by Fan[4] and Risk[5], and including parasitic effects such as energy transfer upconversion (ETU) [6]. In this work we utilized a lat.\% doped Nd:YAG crystal and in the first instance have neglected the higher threshold contribution introduced by ETU, since at the colder temperatures it is not as necessary to have such high inversion densities to achieve sufficient gain. Utilizing the methods proposed by Fan and Risk $[4,5]$ and including the temperature dependence of the Boltzmann factors for the fractional population in the Stark levels of interest, we evaluated the expected performance of the $946 \mathrm{~nm}$ output with reducing temperature of the gain medium, Fig 1. Note these calculations do not account for changes in the actual inter-Stark level cross section as a function of temperature, simply the effective cross section through the changing Boltzmann occupation factors.

\section{Experimental setup}

Our bespoke dewar cryostat is able to dissipate $>100 \mathrm{~W}$ of heat through the interface between the crystal mount and the LN with only a few degree temperature rise. With a capacity to hold $\sim 10 \mathrm{~L}$ of the cryogen, several hours of independent operation can be achieved at this heat loading factor and much more at lower power levels. The dewar and crystal mount are thermally isolated from the external environment via super-insulation and a high-vacuum, the latter provided by a turbo-molecular vacuum pump. A copper heat sink was fixed to the bottom of the LN dewar, on which, a 10mm thick 1.0at.\% Nd:YAG slab, oriented at Brewster's angle, was mounted and held in place with a copper clamping plate. Two $6 \mathrm{~mm}$ thick windows, AR coated for wavelengths around $0.9 \mu \mathrm{m}$, provide optical port holes to access the crystal. The transmission of these windows at the laser wavelength was determined to be $\sim 99.5 \%$ each, accounting for an additional cavity loss of $\sim 2 \%$, on top of the $\sim 1-2 \%$ loss associated with the mirrors and gain medium itself. 
Threshold power

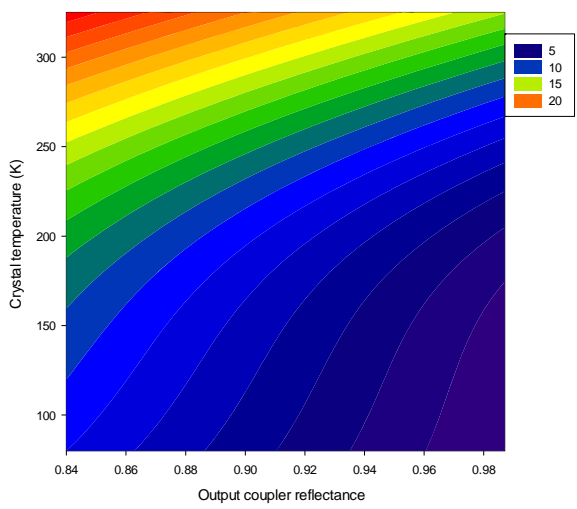

(a)

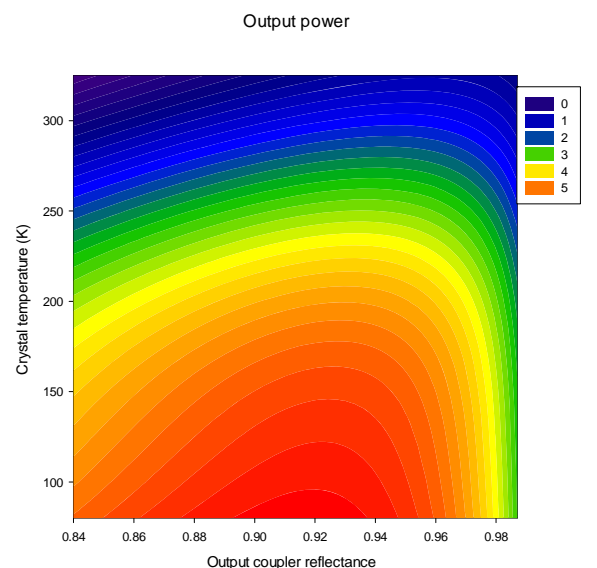

(b)

Fig 1. Theoretical calculations for: (a) threshold power and (b) output power as a function of output coupling and temperature. Assumed cavity characteristics as per the experimental configuration described in section 3 .

A simple three mirror $\mathrm{V}$-cavity was employed for the laser resonator, the crystal pumped by the $808 \mathrm{~nm}$ emission from a fibre-coupled diode laser producing $25 \mathrm{~W}$ from the $400 \mu \mathrm{m}$ core and a 0.22 numerical aperture. A $1.5 \mathrm{x}$ magnification re-imaging telescope produced a minimum pump beam radius of $\sim 300 \mu \mathrm{m}$ midway through the gain medium. The coupling efficiency from the output of the pumping fibre into the crystal was $83 \%$, including the $\sim 10 \%$ reflection loss of the unpolarised pump from the Brewster angled input surface. The room temperature absorption of the $11.5 \mathrm{~mm}$ effective path length through the crystal was $\sim 95 \%$, nominally 3 absorption lengths, that is the crystal was significantly longer than would typically be used for optimal operation of the $946 \mathrm{~nm}$ transition. The pump incoupling high-reflectance (HR) mirror (M1) was position $65 \mathrm{~mm}$ in front of the laser crystal, while the turning mirror (M2) had a $300 \mathrm{~mm}$ radius of curvature and was $240 \mathrm{~mm}$ behind the laser crystal. The final distance between the output coupler (OC) and turning mirror M2 was set between 160-210 mm, see Fig. 2. This setup produced a nominal cold cavity mode radius in the crystal of $\sim 300 \mu \mathrm{m}$.

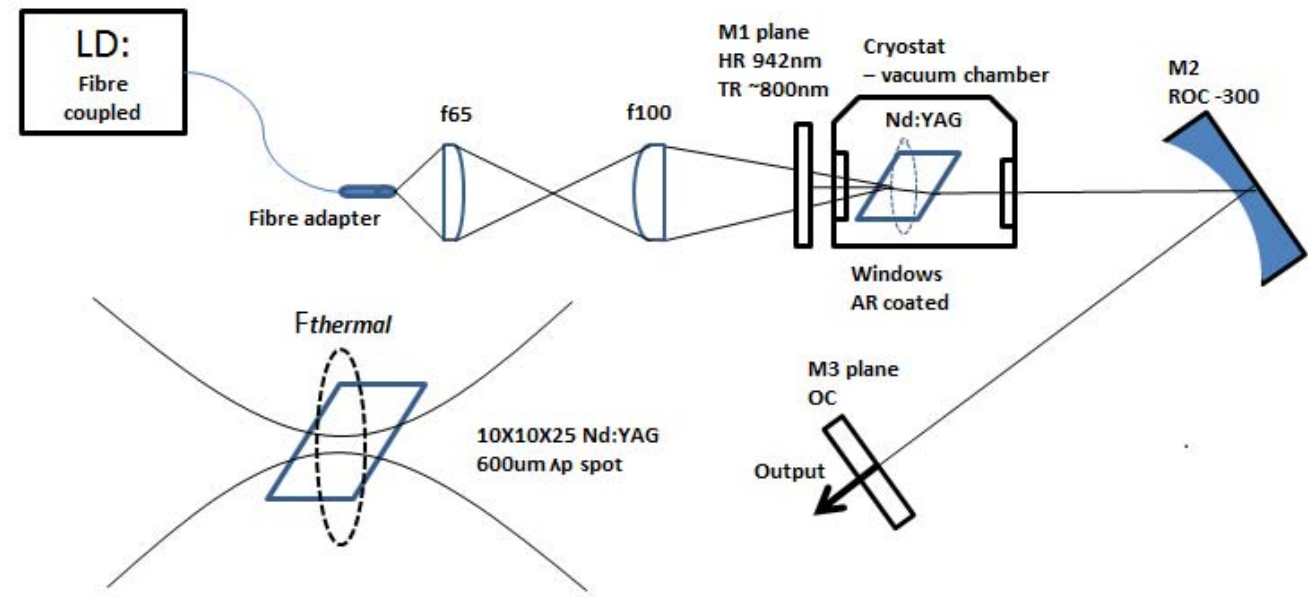

Fig 2. Schematic of laser set-up

\section{Discussion}

When Nd:YAG is cooled to LN temperatures the population of the terminal Stark level of the laser transition is estimated to decrease by $10^{5}$ times with respect to that at $300 \mathrm{~K}$, and therefore introduces essentially zero reabsorption loss, that is, it is practically a four-level laser system. Initial experiments were undertaken with quasi$\mathrm{CW}$ pumping to determine the effects on the temperature dependence of the spectroscopy, independent of thermooptical effects. At full current of $40 \mathrm{~A}$ and $25 \mathrm{~W}$ peak pump power, we turned the diodes on for $2 \mathrm{~ms}$ at a $50 \mathrm{~ms}$ period ( $20 \mathrm{~Hz}$ repetition rate) giving a $4 \%$ duty and $1 \mathrm{~W}$ average power. We did not observe a continual increased in output 
power as would be expected considering the theoretical curves in Fig 1. Rather the average output power reached $190 \mathrm{~mW}(4.75 \mathrm{~W}$ peak) around $187 \mathrm{~K}$, then dropped for temperatures below this falling to $170 \mathrm{~mW}$ (4.25W peak) at LN temperature. The trend in average output power at various dewar temperatures is shown in Fig 3(a), and the decay in performance attributed to a reduced absorption efficiency in the Nd:YAG slab with narrowing spectral features and $\mathrm{a} \sim 3 \mathrm{~nm}$ emission bandwidth for the diode [3]. Unfortunately due to the mounting configuration it was not possible to access the transmitted pump power to verify this; however this will be investigated imminently.

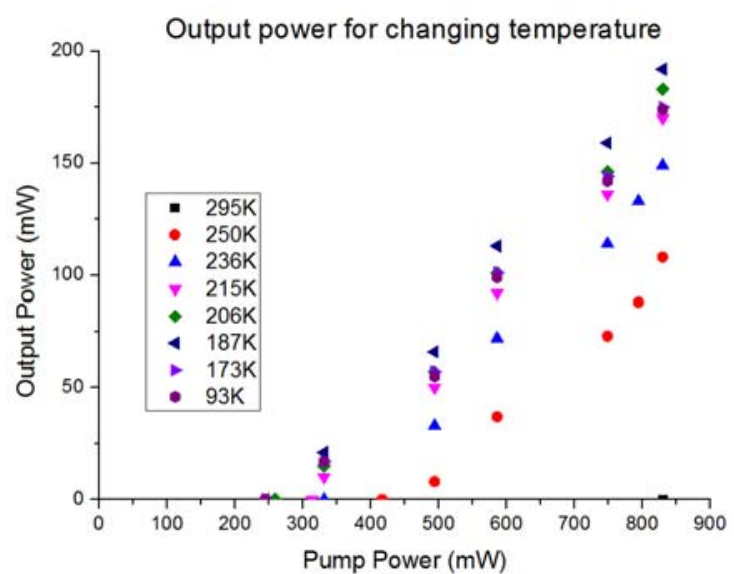

(a)

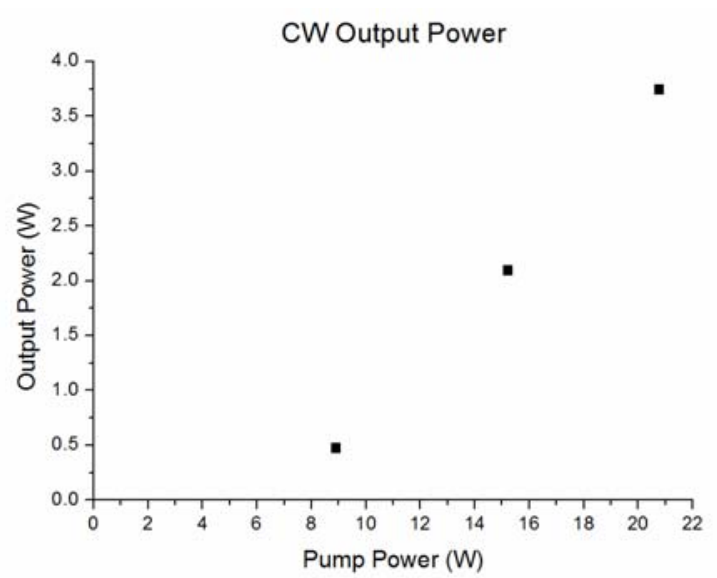

(b)

Fig 3. QCW laser output curve for changing crystal temperature (a), laser output curve for CW operation at LN temperature (b).

The laser was confirmed to be operating on the ${ }^{4} \mathrm{~F}_{3 / 2} \rightarrow{ }^{4} \mathrm{I}_{9 / 2}$ transition at $946 \mathrm{~nm}$ only, via an optical spectrum analyzer. Output power was measured using a Gentec thermopile power meter (UP19K) positioned several hundred millimeters after the OC. We obtained the best performance with a 91\%R output coupling mirror (results shown in Fig. 3) with a $23 \%$ optical-to-optical conversion efficiency at the intermediate temperature of $187 \mathrm{~K}$, with a slope efficiency of $31 \%$. It is clear that below $200 \mathrm{~K}$ the reabsorption losses all but vanish, with the average power lasing threshold converging to $240 \mathrm{~mW}$ ( $6 \mathrm{~W}$ peak).

In a final quick test prior to this submission $\mathrm{CW}$ pumping was tested at a few points with the crystal at $\mathrm{LN}$ temperature, here the maximum power dropped to $3.8 \mathrm{~W}$ with a slope efficiency of $28 \%$, as shown in Fig $3(\mathrm{~b})$. It is anticipated that the $10 \%$ drop in peak power in QCW mode to $\mathrm{CW}$ mode is due to changing thermal lensing conditions in the gain medium and hence a change in mode overlap with the pump distribution, as the diode had been temperature tuned to the same operating wavelength.

\section{Summary}

We have demonstrated the first cryogenically cooled 946nm Nd:YAG operating at the few Watt level. A maximum of $4.8 \mathrm{~W}$ peak power was achieved for $20.7 \mathrm{~W}$ of pump at a temperature of $\sim 190 \mathrm{~K}$, obtained under QCW pumping with a $4 \%$ duty cycle. While for $\mathrm{CW}$ operation $3.8 \mathrm{~W}$ of output power was obtained for $20.7 \mathrm{~W}$ of incident pump, with a slope efficiency of $28 \%$. Further improvements in performance and power scaling considerations will be discussed.

\section{References}

1. D. C. Brown, "The promise of cryogenic solid-state lasers," IEEE J. Sel. Top. Quantum Electron. 11, 587-599 (2005).

2. T. Y. Fan, D. J. Ripin, R. L. Aggarwal, J. R. Ochoa, B. Chann, M. Tilleman, and J. Spitzberg, "Cryogenic Yb3+-doped solid-state lasers," IEEE J. Sel. Top. Quantum Electron. 13, 448-459 (2007).

3. H. Glur, R. Lavi, and T. Graf, "Reduction of thermally induced lenses in Nd : YAG with low temperatures," Ieee Journal of Quantum Electronics 40, 499-504 (2004).

4. T. Y. Fan, and R. L. Byer, "Modeling and CW Operation of a Quasi-3-Level 946 nm Nd:YAG Laser," IEEE Journal of Quantum Electronics 23, 605-612 (1987).

5. W. P. Risk, "Modeling of Longitudinally Pumped Solid-State Lasers Exhibiting Reabsorption Losses," J. Opt. Soc. Am. B-Opt. Phys. $5,1412-1423$ (1988).

6. $\quad$ S. Bjurshagen, R. Koch, and F. Laurell, "Quasi-three-level Nd : YAG laser under diode pumping directly into the emitting level," Opt. Commun. 261, 109-113 (2006). 